\title{
Ginsenoside Rd promotes neurogenesis in rat brain after transient focal cerebral ischemia via activation of PI3K/Akt pathway
}

\author{
Xin-yu LIU ${ }^{1,2, \#, ~ X i n-y u ~ Z H O U ~ 3, ~ \#, ~ J i n-c a i ~ H O U ~}{ }^{1}$, Hua ZHU ${ }^{3}$, Zhong WANG ${ }^{4, *}$, Jian-xun LIU ${ }^{1, *}$, Yong-qiu ZHENG ${ }^{1,3, *}$ \\ ${ }^{1}$ Institute of Basic Medical Sciences of Xiyuan Hospital, China Academy of Chinese Medical Sciences, Beijing 100091, China; ${ }^{2}$ Beijing \\ University of Chinese Medicine, Beijing 100029, China; ${ }^{3}$ Department of Surgery, Davis Heart and Lung Research Institute, Ohio State \\ University Wexner Medical Center, Columbus, OH 43210, USA; ${ }^{4}$ nstitute of Basic Research in Clinical Medicine, China Academy of \\ Chinese Medical Sciences, Beijing 100029, China
}

Aim: To investigate the effects of ginsenoside $\mathrm{Rd}(\mathrm{Rd})$ on neurogenesis in rat brain after ischemia/reperfusion injury (IRI). Methods: Male SD rats were subjected to transient middle cerebral artery occlusion (MCAO) followed by reperfusion. The rats were injected with $\mathrm{Rd}\left(1,2.5\right.$, and $5 \mathrm{mg} \cdot \mathrm{kg}^{-1} \cdot \mathrm{d}^{-1}$, ip) from d 1 to d 3 after MCAO, and with BrdU (50 $\mathrm{mg}^{\mathrm{kg}} \mathrm{kg}^{-1} \cdot \mathrm{d}^{-1}$, ip) from d 3 to d 6 , then sacrificed on $7 \mathrm{~d}$. The infarct size and neurological scores were assessed. Neurogenesis in the brains was detected by BrdU, DCX, Nestin, and GFAP immunohistochemistry staining. PC12 cells subjected to OGD/reperfusion were used as an in vitro model of brain ischemia. VEGF and BDNF levels were assessed with ELISA, and Akt and ERK phosphorylation was measured using Western blotting. Results: Rd administration dose-dependently decreased the infarct size and neurological scores in the rats with IRI. The high dose of $\mathrm{Rd}\left(5 \mathrm{mg} \cdot \mathrm{kg}^{-1} \cdot \mathrm{d}^{-1}\right)$ significantly increased Akt phosphorylation in ipsilateral hemisphere, and markedly increased the number of BrdU/ DCX and Nestin/GFAP double-positive cells in ischemic area, which was partially blocked by co-administration of the PI3 kinase inhibitor LY294002. Treatment with Rd (25, 50, and $100 \mu \mathrm{mol} / \mathrm{L})$ during reperfusion significantly increased the expression of VEGF and BDNF in PC12 cells with IRI. Furthermore, treatment with Rd dose-dependently increased the phosphorylation of Akt and ERK, and significantly decreased PC12 cell apoptosis, which were blocked by co-application of LY294002.

Conclusion: Rd not only attenuates ischemia/reperfusion injury in rat brain, but also promotes neurogenesis via increasing VEGF and BDNF expression and activating the PI3K/Akt and ERK1/2 pathways.

Keywords: ginsenoside Rd; stroke; cerebral ischemia; neurogenesis; VEGF; BDNF; Akt; ERK; LY294002

Acta Pharmacologica Sinica (2015) 36: 421-428; doi: 10.1038/aps.2014.156; published online 16 Mar 2015

\section{Introduction}

Recently, researchers have shown keen interest in neurogenesis after stroke. New neurons and glia are continuously generated by neural stem cells (NSCs) in the subventricular zone (SVZ) and subgranular zone (SGZ) of the adult brain during the restoration of nerve function, a process called neurogenesis. According to several studies, neurogenesis occurs in response to IRI, causing brain structure remodeling and self-repair ${ }^{[1,2]}$. A middle cerebral artery occlusion (MCAO) can activate the proliferation, migration and differentiation of NSCs and promote their integration in existing nerve tissues ${ }^{[3]}$.

\footnotetext{
"These authors contributed equally to this work.

* To whom correspondence should be addressed.

E-mail yongqiuzheng@sina.com (Yong-qiu ZHENG); liujx0321@sina.com (Jian-xun LIU); zhonw@vip.sina.com (Zhong WANG)

Received 2014-08-27 Accepted 2014-12-20
}

In addition, stroke induced neurogenesis reportedly still exists in aging rats, although the capacity for neurogenesis in young adults is higher ${ }^{[4]}$. In recent years, neurogenic Chinese herbal medicines have generated great interest among researchers, particularly regarding their utility for rehabilitating the penumbra after ischemia/reperfusion injury (IRI). Although much work has been done, more studies are still needed to ascertain the mechanisms of function of these Chinese herbal medicines after stroke.

Ginsenosides are the most active components of ginseng; more than 40 ginsenosides, including $\mathrm{Rd}$, have been identified and isolated from this root ${ }^{[5]}$. Numerous studies have demonstrated that $\mathrm{Rd}$ is beneficial in the treatment of ischemic stroke. $\mathrm{Rd}$ can protect neurons from oxidative stress injury caused by hydrogen peroxide and oxygen glucose deprivation $(\mathrm{OGD})^{[6]}$. In addition, $\mathrm{Rd}$ can cross the intact blood-brain barrier and exert protective effects during both transient and 
permanent $\mathrm{MCAO}$ in rats ${ }^{[7]}$. However, although this traditional Chinese medicine has long been used to strengthen the body's resistance to pathogenic factors, the beneficial effects of ginsenosides on cerebral self-remodeling after ischemia have not been fully elucidated. Thus, in this study, we aim to verify whether Rd, an active ingredient of ginseng, promotes neurogenesis after MCAO.

Several cytokines are involved in neurogenesis. VEGF is the strongest growth factor that promotes SVZ neurogenesis and neural migration. According to some studies, VEGF overexpression reduces infarct volume and improves post-ischemic motor function, which may contribute to brain recovery and repair $^{[8]}$. Furthermore, vascular endothelial cells secrete VEGF to promote the proliferation of NSCs ${ }^{[9]}$. BDNF is a member of the nerve growth factor family and is released after hypoxia or ischemia to protect the brain from injury. BDNF plays an important role in narrowing infarct size, protecting penumbral neurons and repairing broken neurons ${ }^{[10]}$. This factor can stimulate adult neurogenesis and enhance the appearance and migration of new neurons in the SVZ and dentate gyrus ${ }^{[11]}$. Notably, the expression of both VEGF and BDNF is regulated by PI3K/ $\mathrm{Akt}^{[12]}$ and ERK1/2 pathways ${ }^{[13]}$, and Rd can activate these pathways ${ }^{[14]}$. Therefore, in the present study, we demonstrate that the PI3K/Akt and ERK1/2 pathways are involved in the protective effects of Rd. Further, this is the first study, to our knowledge, to examine the potential link between the ginsenosides and neurogenesis in response to cerebral ischemia/reperfusion.

\section{Materials and methods}

Animals

Adult male Sprague-Dawley (SD) rats (220-240 g), provided by the Beijing Vital River Company, were housed in the laboratory animal room and maintained at $25 \pm 1^{\circ} \mathrm{C}$ with $65 \% \pm 5 \%$ humidity on a 12-h light/dark cycle (lights on from 7:30 to 19:30) for at least 1 week before the start of the experiments. The animals were given food and water ad libitum. All experimental protocols described in this study were approved by the Ethics Review Committee for Animal Experimentation of Xiyuan Hospital, at the China Academy of Chinese Medical Sciences.

\section{Drugs}

The Rd (white powder, purity of ginseng >95\%) used in the present study was kindly donated by the School of Medicine, Beijing University. Rd was dissolved in dimethyl sulfoxide (DMSO) for the in vitro experiments and was dissolved in $0.9 \%$ physiological saline containing 5\% CMC-Na for the in vivo experiments.

\section{Materials and reagents}

The mouse anti-BrdU antibody was purchased from Santa Cruz Biotechnology (Santa Cruz, CA, USA), while the rabbit anti-DCX, rabbit anti-GFAP and mouse anti-Nestin antibodies were obtained from Cell Signaling Technology (Danvers, MA, USA). Anti-mouse IgG-fluorescein isothiocyanate (FITC) and anti-rabbit IgG-Cy3 antibodies were purchased from SigmaAldrich (St Louis, MO, USA). The Dulbecco's modified Eagle's medium (DMEM) and the fetal bovine serum (FBS) used for cell culture and treatments were purchased from Gibco Co (CA, USA). Horseradish peroxidase-conjugated AffiniPure goat anti-mouse and anti-rabbit IgG were obtained from Sigma-Aldrich (St Louis, MO, USA). The rabbit anti-ERK-1/2, mouse anti-phospho-ERK-1/2 (pThr202/pTyr204), mouse anti-Akt and rabbit anti-phospho-Akt (pThr324/pSer437) antibodies were purchased from Sigma (St Louis, MO, USA), while the PI3 Kinase inhibitor, LY294002, was purchased from Cell Signaling Technology (USA). The VEGF and BDNF levels were determined by enzyme-linked immunosorbent assays (ELISA), using kits produced by R\&D Systems (Minneapolis, MN, USA). Akt and ERK were electrophoretically transferred to polyvinylidene fluoride (PVDF) microporous membranes (Merck Millipore, Billerica, MA, USA) and then visualized using the Enhanced SuperSignal West femto kit from Pierce (Rockford, IL, USA). The Labotect $\mathrm{CO}_{2}$-Incubator was a product of SANYO Electric Co Ltd (Los Angeles, CA, USA). The OGD device was built by the experimenters.

\section{Drug treatment}

Before the surgical operation, the male SD rats were randomly divided into 4 groups (10 animals/group): the sham group, the IRI group, the IRI+Rd (5 mg/ $\mathrm{kg}$ ) group, and the IRI+Rd (5 $\mathrm{mg} / \mathrm{kg})+\mathrm{LY} 294002(10 \mathrm{mg} / \mathrm{kg})$ group. From d 1 to $\mathrm{d} 3$ after $\mathrm{MCAO}$, the rats were given $\mathrm{Rd}(5 \mathrm{mg} / \mathrm{kg})$ daily by intraperitoneal injection (ip). The LY294002 (10 mg/ kg, ip) was injected $30 \mathrm{~min}$ before MCAO and again $48 \mathrm{~h}$ after reperfusion. The sham and MCAO controls were given an equal volume of the vehicle $(0.9 \%$ physiological saline contained $5 \%$ CMC-Na).

To assess infarct size in vivo, the male SD rats were randomly divided into 5 groups (6 animals/group): the sham group, the IRI group, the IRI+Rd ( $5 \mathrm{mg} / \mathrm{kg}$ ) group, the IRI+Rd $(2.5 \mathrm{mg} / \mathrm{kg})$ group and the IRI+Rd $(1 \mathrm{mg} / \mathrm{kg})$ group. From $\mathrm{d} 1$ through $\mathrm{d} 3$ after MCAO, the rats were given $\mathrm{Rd}$ or vehicle by intraperitoneal injection (ip).

To detect Akt phosphorylation in vivo, the male SD rats were randomly divided into 5 groups ( 3 animals/group), as mentioned above. Both $1 \mathrm{~h}$ before and $12 \mathrm{~h}$ after reperfusion, the rats were given either $\mathrm{Rd}$ or the vehicle by intraperitoneal injection (ip).

\section{Transient MCAO}

Rats with physiological variables within normal ranges were subjected to transient focal cerebral ischemia induced by right MCAO as previously described ${ }^{[15]}$. After 90 min of MCAO, the suture was pulled back until the tip reached the suture around the internal carotid artery (ICA) to restore blood flow (reperfusion). After the wound was closed, the animals were allowed to recover from anesthesia before they were returned to their home cages.

\section{BrdU labeling and tissue preparation}

To label dividing cells, intraperitoneal injections of BrdU (50 
$\mathrm{mg} / \mathrm{kg}$ ) were given once daily after MCAO from d 3 to d 6 (5 animals/group).

Rats were sacrificed $7 \mathrm{~d}$ after MCAO by an overdose of $3.5 \%$ chloral hydrate $(0.1 \mathrm{~mL} / \mathrm{kg})$ and transcardially perfused with $0.9 \%$ saline solution followed by $4 \%$ ice-cold phosphatebuffered paraformaldehyde (PFA, $40 \mathrm{~g} / \mathrm{L}$ ). The brains were removed, postfixed overnight, and equilibrated in phosphatebuffered $30 \%$ sucrose $(200 \mathrm{~g} / \mathrm{L})$. Coronal sections at 1.0 to 0.2 $\mathrm{mm}$ from bregma were used for immunohistochemical staining and were cut on a freezing microtome (Leica CM3000, Germany) at a thickness of $25 \mu \mathrm{m}$.

\section{Immunohistochemistry and immunofluorescent staining}

Frozen sections were double-stained by the colocalization of phenotypic markers using the following primary antibodies (and their concentrations): rabbit polyclonal anti-Doublecortin (DCX) to label neural progenitor cells (1:100); mouse monoclonal anti-BrdU to label dividing cells (1:100); rabbit antiglial fibrillary acidic protein (GFAP), to label mature astrocytes (1:100); mouse polyclonal anti-Nestin to label neuronal stem cells (1:100). Secondary antibodies: anti-mouse, rabbit and goat IgG-FITC and IgG-Cy3 (1:50; Chemicon). Confocal images were taken using a Zeiss LSM-510 microscope.

\section{Measurement of infarct volume}

To assess infarct size in vivo, the rats were deeply anesthetized with $3.5 \%$ chloral hydrate and then decapitated $7 \mathrm{~d}$ after reperfusion, after which the whole brains were rapidly removed. Coronal sections ( $n=6$ for each group) were cut into 2-mm slices and stained with standard 2\% 2,3,5-triphenyltetrazolium chloride (TTC, Sigma-Aldrich) for $10 \mathrm{~min}$ at $37^{\circ} \mathrm{C}$, followed by overnight immersion in $4 \%$ formalin. Infarct volume, expressed as a percentage of the whole-brain volume, was measured by an image processing and analysis system (1.25×objective, Q570IW; Leica, Wetzlar, Germany) and was calculated by integration of the infarct area on each brain section along the rostral-caudal axis.

\section{Assessment of neurological deficit score}

The neurological deficit score was assessed $24 \mathrm{~h}$ and $7 \mathrm{~d}$ after reperfusion. Each rat was scored by 2 examiners who were kept unaware of the identity of the rat and the treatment protocol. The following neurological deficit scoring (NDS) system was used: 0 , no motor deficits (normal); 1 , forelimb weakness and torso turning to the ipsilateral side when held by tail (mild); 2, circling to the contralateral side but normal posture at rest (moderate); 3 , unable to bear weight on the affected side at rest (severe); and 4, no spontaneous locomotor activity or barrel rolling (critical). If no deficit was observed $2 \mathrm{~h}$ recovering from anesthesia, the animal was removed from further study.

\section{Cell culture and OGD model}

PC12 cells were kindly donated by the School of Medicine, Beijing University. These cells were grown in DMEM with high glucose containing $10 \% \mathrm{FBS}, 100 \mathrm{U} / \mathrm{mL}$ penicillin and
$100 \mathrm{U} / \mathrm{mL}$ streptomycin in a humid chamber at $37^{\circ} \mathrm{C}$ under $5 \% \mathrm{CO}_{2}$. The OGD insult, followed by reoxygenation, is used as an in vitro model of cerebral ischemia.

PC12 cells were seeded at $1 \times 10^{6}$ cells/well in 6-well plates, incubated overnight and then washed twice with glucose-free Earle's balanced salt solution (glucose-free OGD g/ $\mathrm{L}: \mathrm{NaCl}$ 6.8, $\mathrm{KCl} 0.4, \mathrm{CaCl}_{2} \cdot 2 \mathrm{H}_{2} \mathrm{O} 0.264, \mathrm{MgSO}_{4} \cdot 7 \mathrm{H}_{2} \mathrm{O} 0.2, \mathrm{NaH}_{2} \mathrm{PO}_{4} \cdot 2 \mathrm{H}_{2} \mathrm{O}$ $0.156, \mathrm{NaHCO}_{3} 2.2$ ). The cells were divided into different groups: the control group, the OGD model group, the OGD+low dose Rd group ( $25 \mu \mathrm{mol} / \mathrm{L})$, the OGD+middle dose $\mathrm{Rd}$ group ( $50 \mathrm{\mu mol} / \mathrm{L})$, the OGD+high dose $\mathrm{Rd}$ group $(100 \mu \mathrm{mol} / \mathrm{L})$ and the $\mathrm{Rd}(50 \mu \mathrm{mol} / \mathrm{L})+\mathrm{LY} 294002(10 \mu \mathrm{mol} / \mathrm{L})$ group. To induce OGD, the culture media was replaced with glucose-free OGD and the cells were incubated in a modified OGD device ventilated with a $95 \% \mathrm{~N}_{2}-5 \% \mathrm{CO}_{2}$ gas mixture for $30 \mathrm{~min}$. Then, the cells were kept in the chamber at $37^{\circ} \mathrm{C}$ for $4 \mathrm{~h}$. Next, normal serum-free DMEM $(\mathrm{NaCl} 6.8, \mathrm{KCl} 0.4$, $\mathrm{CaCl}_{2} \cdot 2 \mathrm{H}_{2} \mathrm{O} 0.264, \mathrm{MgSO}_{4} \cdot 7 \mathrm{H}_{2} \mathrm{O} 0.2, \mathrm{NaH}_{2} \mathrm{PO}_{4} \cdot 2 \mathrm{H}_{2} \mathrm{O} 0.156$, $\mathrm{NaHCO}_{3} 2.2$, glucose 1.0) was replaced for another $6 \mathrm{~h}$ as the OGD reperfusion model ${ }^{[16]}$. The control culture was subjected to the same experimental procedures without exposure to the glucose-free DMEM medium. The different concentrations of $\mathrm{Rd}(25,50$, and $100 \mu \mathrm{mol} / \mathrm{L} /$ dissolved in DMSO to $10 \mathrm{mmol} / \mathrm{L})$ and LY294002 (10 $\mathrm{mmol} / \mathrm{L} /$ dissolved in DMSO to $10 \mathrm{mmol} / \mathrm{L}$ ) were added after the $4 \mathrm{~h}$ OGD treatment and throughout the OGD-reperfusion within the standard medium. Then, the supernatants (SN) were collected for cytokine detection, and the cells were collected for apoptosis assays or Western blotting detection.

\section{VEGF and BDNF assay}

The supernatants (SN) containing VEGF and BDNF were centrifuged $\left(1000 \times g, 10 \mathrm{~min}, 4^{\circ} \mathrm{C}\right)$ and the concentrations of VEGF and BDNF in the supernatants were measured with ELISA kits, according to the manufacturers' directions. Sample and standard dilutions were made with the experimental media, and the results are expressed as the means $\pm \mathrm{SD}^{[9]}$.

\section{Western blotting}

Protein from PC12 cells was collected and diluted to $0.5 \mathrm{mg}$ protein/mL for the measurement of ERK and the phosphorylation of Akt. In vivo, the rats were deeply anesthetized with $3.5 \%$ chloral hydrate and then decapitated $24 \mathrm{~h}$ after reperfusion, after which the whole brains were rapidly removed. Cortical sections at 1.0 to $2.0 \mathrm{~mm}$ from the infarcts were used for Western blotting analysis. The total protein content was determined with BSA as a standard using the Bradford assay. Protein samples $(20 \mu \mathrm{g} / \mathrm{lane})$ were separated by sodium dodecyl sulfate-polyacrylamide gel electrophoresis and were transferred to PVDF membranes (Millipore, Billerica, MA, USA). The blots were stained for phosphorylated Akt (Ser473), total Akt, phosphorylated ERK1/2 (pTyr204) and total ERK1/2. The stained blots were visualized by enhanced chemiluminescence using the SuperSignal West femto maximum sensitivity substrate (Pierce, Rockford, IL, USA). The Bio-Rad Image $\mathrm{Lab}^{\mathrm{TM}}$ Version 3.0 software was used to quantify every blot, 
and their mean densitometric $\times$ area values are depicted as bar graphs.

\section{Analysis of apoptosis}

The cells were collected and double-stained with FITCconjugated Annexin-V $(25 \mu \mathrm{g} / \mathrm{mL})$ and propidium iodide (PI) $(50 \mu \mathrm{g} / \mathrm{mL}) 142$ (Beckman Coulter) for $20 \mathrm{~min}$ in the dark. Then the cells were collected on an Epic XL flow cytometer equipped with a 488-nm 143 argon laser and analyzed using the Expo32 flow cytometry software (Beckman Coulter).

\section{Statistical analysis}

All data are expressed as the mean \pm SD. The statistical analyses were performed using SPSS, version 11.0. ANOVA or nonparametric tests were used for the statistical analyses. $P<0.05$ was considered statistically significant.

\section{Results}

Effects of Rd on VEGF and BDNF concentrations in PC12 cells treated with $\mathrm{OGD} /$ reperfusion

VEGF is one of the growth factors, and BDNF is one of the neurotrophic factors that are related to neurogenesis. We detected the VEGF and BDNF levels by analyzing the supernatants from PC12 cells after OGD. As expected, the expression of VEGF and BDNF was enhanced after OGD/reperfusion as compared with the control group (Figure 1). After OGD, the PC12 cells were treated with different doses of Rd $(25,50$, or
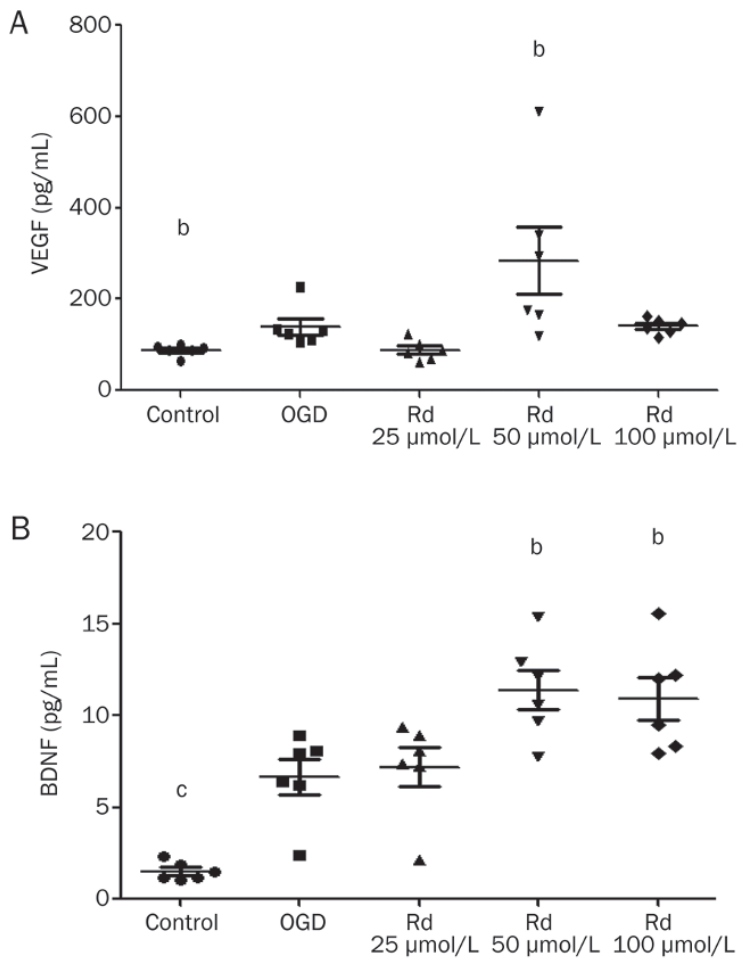

Figure 1. Contents of VEGF/BDNF in CM of PC12 cells. (A) Quantitative data from each group. ANOVA was used for statistical analysis. (B) Quantitative data from each group. ANOVA was used for statistical analysis. Data are mean \pm SD. $n=6 .{ }^{\mathrm{b}} P<0.05,{ }^{\mathrm{C}} P<0.01$ vs OGD.
$100 \mu \mathrm{mol} / \mathrm{L}$ ). The results showed that the VEGF expression was significantly higher in the $50 \mu \mathrm{mol} / \mathrm{L} \mathrm{Rd}$ treatment group compared with the control $(P<0.05$; Figure $1 \mathrm{~A})$ and that the BDNF levels were significantly higher in both the 50 and 100 $\mu \mathrm{mol} / \mathrm{L} \mathrm{Rd}$ treatment groups than in the control $(P<0.05$; Figure $1 \mathrm{~B})$. Thus, $\mathrm{Rd}$ treatment strengthened the expression of VEGF and BDNF after OGD/reperfusion.

\section{Effects of Rd on Akt and ERK signaling activation in PC12 cells treated with $O G D /$ reperfusion}

It has been shown that activation of Akt and ERK pathways, which can be detected by the phosphorylation of these two proteins, mediates neurogenesis in PC12 cells ${ }^{[17]}$ and in human umbilical vein endothelial cells ${ }^{[18]}$. Therefore, we investigated the effects of Rd on the phosphorylation of Akt and ERK1/2. In the current study, increased phosphorylation of Akt and ERK was detected in PC12 cells treated with OGD/reperfusion, and Rd (50 and $100 \mu \mathrm{mol} / \mathrm{L})$ significantly $(P<0.05)$ amplified the Akt and ERK phosphorylation triggered by OGD/ reperfusion (Figure 2). These results suggest that the modu-

A
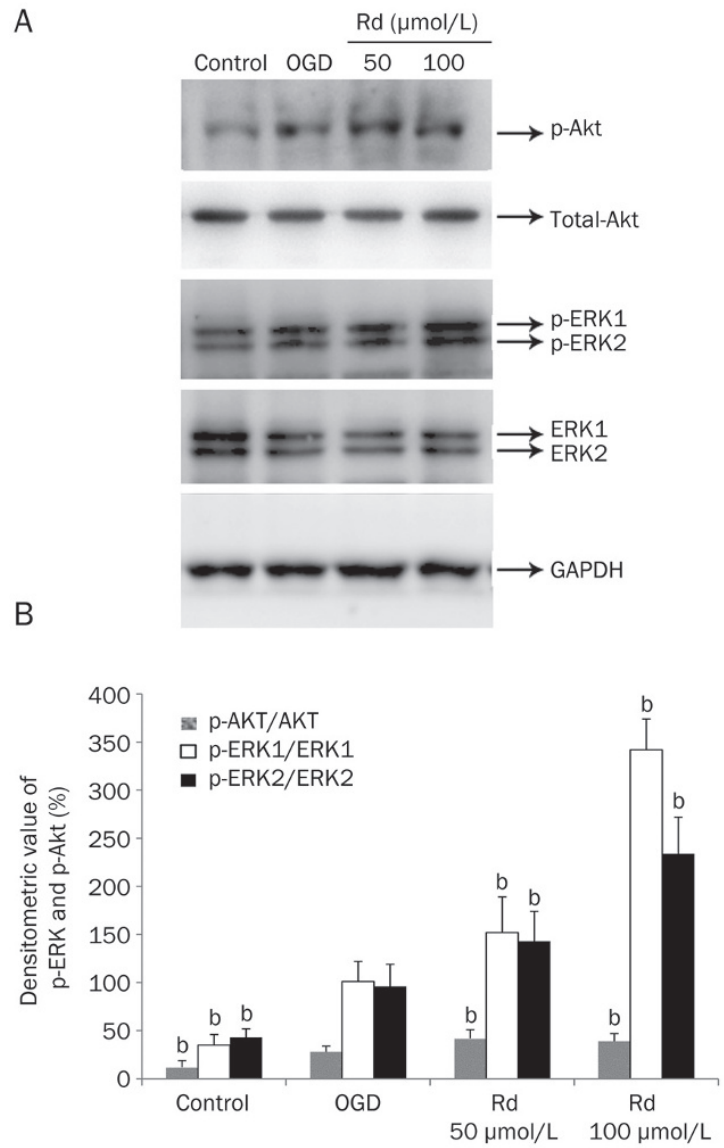

Figure 2. Effects of Rd on phosphorylation of Akt and ERK after OGD. Increased phosphorylation of Akt and ERK proteins in PC12 cells treated by OGD+Rd. An antibody for GAPDH was used to show equal protein loading. (A) Western blot analysis of the phosphorylated forms of Akt (p-Akt-ser473); total Akt; phosphorylated ERK1/2 (p-ERK1/2-tyr204) or total ERK1/2. (B) Bar graphs show quantitative evaluation. Data are mean \pm SD. $n=3 .{ }^{\mathrm{b}} P<0.05$ vs OGD. 
lation of PI3K/Akt and ERK1/2 signaling may be one of the pathways by which Rd exerts its effects after OGD.

\section{Disruption of the PI3K/Akt pathway reversed the biological} effects of RD on PC12 apoptosis

To determine whether the PI3K/Akt pathway is necessary for the observed effects of $\mathrm{Rd}$, we evaluated the protective effects of Rd in the presence of LY294002, an inhibitor of PI3K. Figure 3 shows that the level of phosphorylated Akt was significantly decreased by LY294002 (10 $\mathrm{mmol} / \mathrm{L})$. Compared with the sham group, OGD decreased the cell viability significantly $(P<0.05)$, which was reversed by $50 \mu \mathrm{mol} / \mathrm{L}$ Rd (Figure 4A-4C). Further studies showed that co-treatment with Rd (50 $\mathrm{mmol} / \mathrm{L})$ and LY294002 $(10 \mu \mathrm{mol} / \mathrm{L})$ significantly $(P<0.05)$ increased the apoptotic population compared with the cells treated with OGD+Rd (Figure 4D). These results demonstrate that $\mathrm{Rd}$ opposes apoptosis by activating the PI3K/Akt pathway.

A

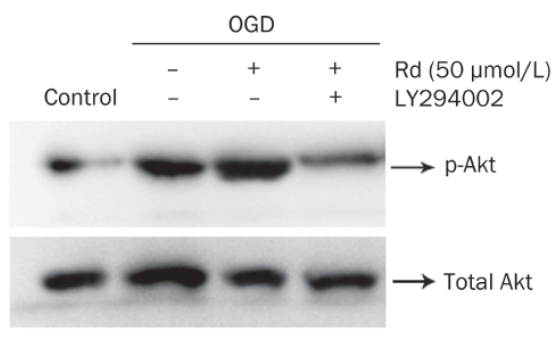

$\mathrm{B}$

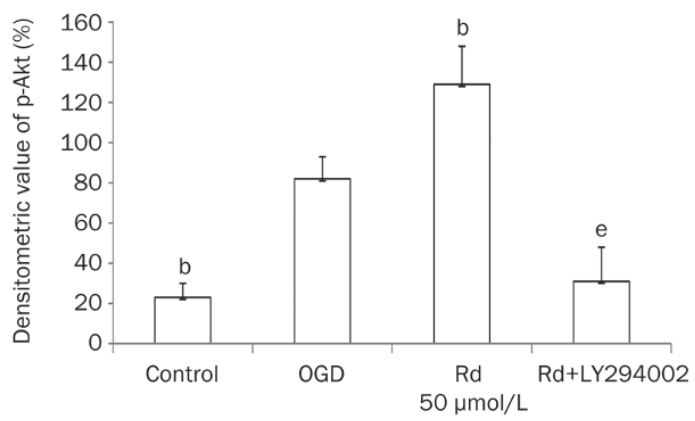

Figure 3. Effects of LY294002 on the PI3K/Akt pathway. (A) PC12 cells were treated with $\mathrm{Rd}(50 \mu \mathrm{mol} / \mathrm{L})$ or LY294002 (10 $\mu \mathrm{mol} / \mathrm{L})$ for $6 \mathrm{~h}$ after OGD, and the expression of Akt was verified by Western blotting. (B) Bar graphs show quantitative evaluation. Data are the mean \pm SD. $n=3$. ${ }^{\mathrm{b}} P<0.05$ vs OGD. ${ }^{\mathrm{e}} P<0.05$ vs OGD+Rd.

\section{Effects of Rd on cerebral infarction and Akt phosphorylation}

Transient focal ischemia for 90 min caused infarction at $7 \mathrm{~d}$ post-reperfusion. Examination of serial brain sections showed that Rd treatment (5 and $2.5 \mathrm{mg} / \mathrm{kg}$ ) significantly decreased the infarct volume compared with the I/ R group $7 \mathrm{~d}$ after focal ischemia/reperfusion. The neurological deficit scores decreased in the I/ $\mathrm{R}$ group, and significant differences were found at $24 \mathrm{~h}$ and $7 \mathrm{~d}$ after reperfusion compared with the $\mathrm{Rd}$
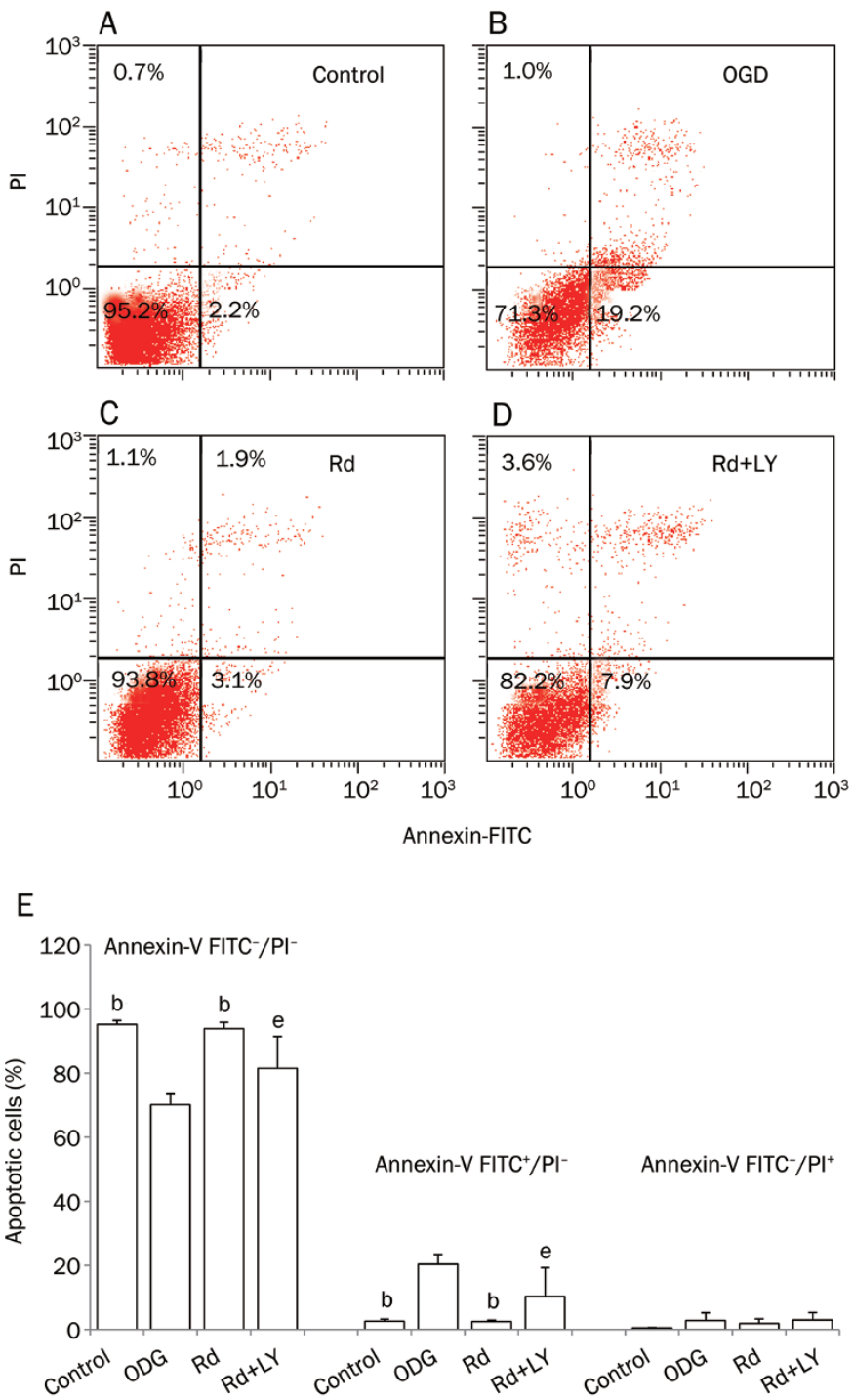

Figure 4. Effects of Rd on apoptosis of PC12 cells after OGD. (A-D) Apoptosis was analyzed by flow cytometry following Pl/Annexin V-FITC double-staining. (E) The percentages of apoptotic cells are indicated. $n=6 .{ }^{\mathrm{b}} P<0.05$ vs OGD. ${ }^{\mathrm{e}} P<0.05$ vs $\mathrm{OGD}+\mathrm{Rd}$.

(5 mg/kg) treatment group (Figure 5). These findings indicate that $\mathrm{Rd}$ protects against ischemic brain injury. Additional studies indicated that $\mathrm{Rd}(5 \mathrm{mg} / \mathrm{kg})$ significantly increased Akt phosphorylation $24 \mathrm{~h}$ after focal ischemia/reperfusion (Figure 6).

\section{Effects of Rd on neurogenesis}

Next, we wondered whether Rd promoted neurogenesis after $\mathrm{MCAO} /$ reperfusion through the PI3K/Akt pathway, so we observed the number of cells $7 \mathrm{~d}$ post-reperfusion. The numbers of $\mathrm{Nestin}^{+} / \mathrm{GFAP}^{+}$cells increased in the striatum and of $\mathrm{BrdU}^{+} / \mathrm{DCX}^{+}$cells increased in the dentate gyrus (DG) in areas surrounding the ischemic core $7 \mathrm{~d}$ after $\mathrm{MCAO} /$ reperfusion, and these increases were promoted by $\mathrm{Rd}(5 \mathrm{mg} / \mathrm{kg}, P<0.05)$. Furthermore, the beneficial effects of $\mathrm{Rd}$ in promoting the 


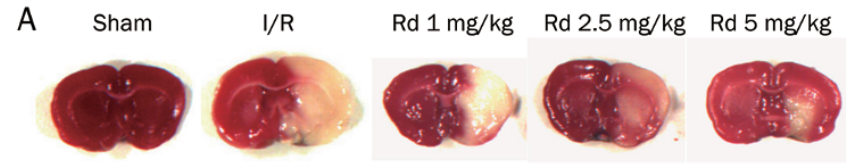

B

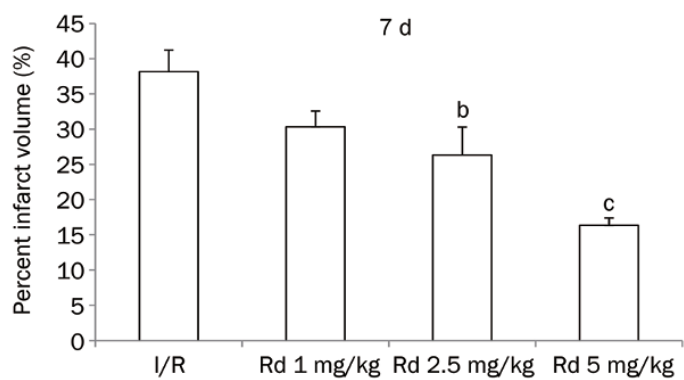

C

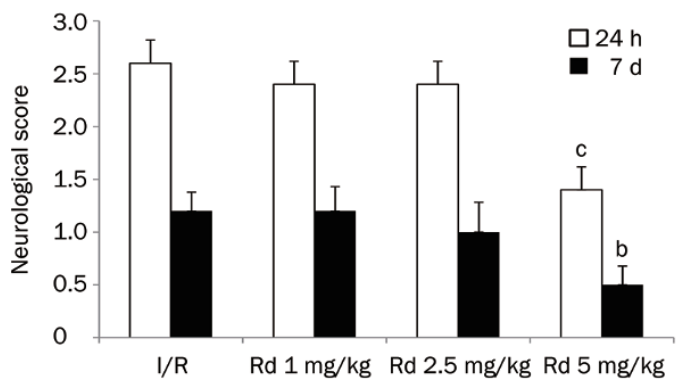

Figure 5. Infarct volume and neurological scores. (A) Representative TTC staining of the cerebral infarct in comparable sections of rat brain, $7 \mathrm{~d}$ after ischemia. (B) Quantification of infarct volume. (C) Neurological scores. Mean \pm SD. $n=6 .{ }^{b} P<0.05,{ }^{c} P<0.01$ vs I/R group.

newborn neural progenitor cells $\left(\mathrm{BrdU}^{+} / \mathrm{DCX}^{+}\right)$and newborn astrocytes $\left(\mathrm{Nestin}^{+} / \mathrm{GFAP}^{+}\right.$) were inhibited by LY294002 $(P<0.05, P<0.01$, Figure 7$)$.

\section{Discussion}

After IRI/reperfusion, Rd promotes neurogenesis in response to transient MCAO, increases the expression of VEGF and BDNF, and activates the PI3K/Akt and ERK1/2 pathways. These findings suggest that Rd-mediated neurogenic mechanisms may be a target for treatments aimed at repairing ischemic injury after stroke in humans.

The traditional view held that the central nervous system (CNS) of adult mammals was incapable of repair after transient MCAO. This opinion was demolished by research that revealed the NSCs could differentiate into three main cells of the $\mathrm{CNS}^{[1]}$. The injury caused by ischemia increases the migration of neuronal precursors, and a sequence and combination of molecular signals does exist that stimulates neurogenesis in the adult cerebral cortex. According to some studies, neurogenesis is induced by $\mathrm{MCAO}$ in several regions, including the SVZ, neocortex, SEZ and striatum ${ }^{[19]}$. The NSCs and proliferating progenitors seem to decline in the aged SVZ and striatum, which might be caused by the apoptosis of NSCs ${ }^{[4]}$. In our study, we investigated neurogenesis in the ipsilateral
A
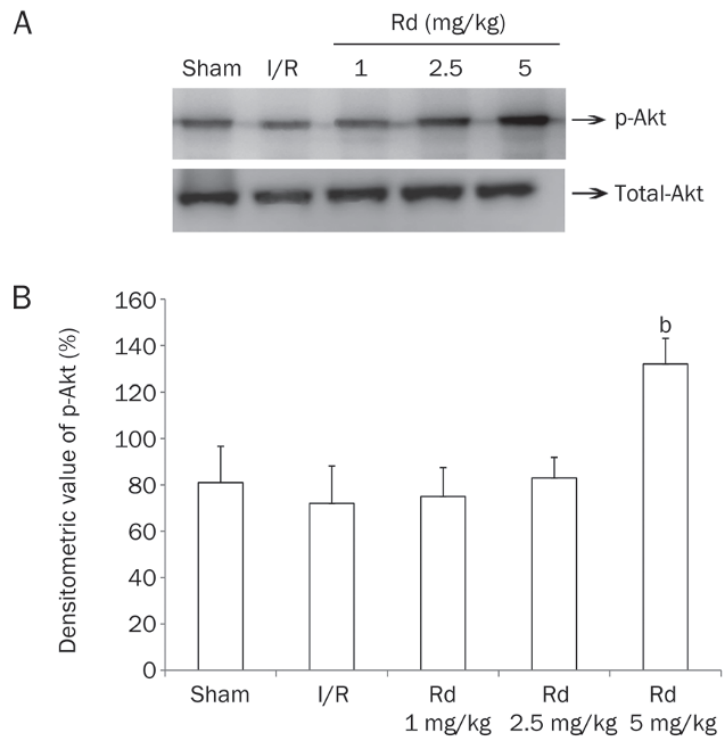

Figure 6. Akt phosphorylation in ipsilateral hemisphere. (A) Western blot analysis of Akt expression in the ipsilateral hemisphere $24 \mathrm{~h}$ after reperfusion. (B) Bar graphs show quantitative evaluation of phospho-Akt/ Akt expression. Means \pm SD. $n=3$. ${ }^{b} P<0.05$ compared with I/R group.

striatum and DG after MCAO treated with Rd. Rd is already recognized as effective and safe for the treatment of acute ischemic stroke ${ }^{[14]}$. Our results support the view that the protective effects of Rd against transient MCAO occur through the promotion of neurogenesis (Figure 7).

VEGF, an endothelial cell (ECs) mitogen, promotes the proliferation of ECs, plays a role in neurotrophy and neuroprotection and improves learning and memory in rats ${ }^{[20]}$. Furthermore, VEGF reduces infarct size and improves the functional outcome when administered within hours to days after MCAO in rodents. Most important of all, VEGF increases the proliferation of neuronal precursors in the SVZ and their migration to the ischemic penumbra ${ }^{[8]}$. BDNF, as one of important regulators of adult neurogenesis, has multiple effects on neurogenesis. This factor can promote the survival, growth and differentiation of neurons, and it can maintain their function and regeneration. In addition, BDNF plays an important role in enhancing synaptic plasticity in long-term potentiation, learning and memory ${ }^{[21]}$. These findings are consistent with the observation that BDNF treatment improves long-term functional neurological outcomes by inducing neurogenesis, parallel with the cellular proliferation and survival of NSCs ${ }^{[11]}$. In the present study, we thus demonstrated that Rd-mediated promotion of neurogenesis increased the levels of VEGF and BDNF in the CM of PC12 cells (Figure 1). The PI3K/ Akt and ERK1/2 pathways can modulate the activation of transcription factors and gene expression to influence neurogenesis ${ }^{[13]}$. Prior studies have shown that the VEGF- and BDNF-mediated downstream signaling cascades for the survival of NSCs, such as the PI3K/Akt or ERK pathways, are enchained upon activation of the tyrosine kinase receptor (TrkB) and VEGF receptor 2 , respectively ${ }^{[11]}$. Based on these findings, we speculated that 
A
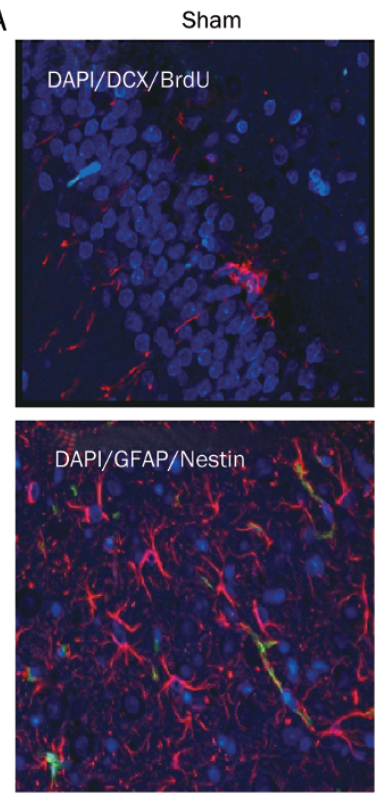

B
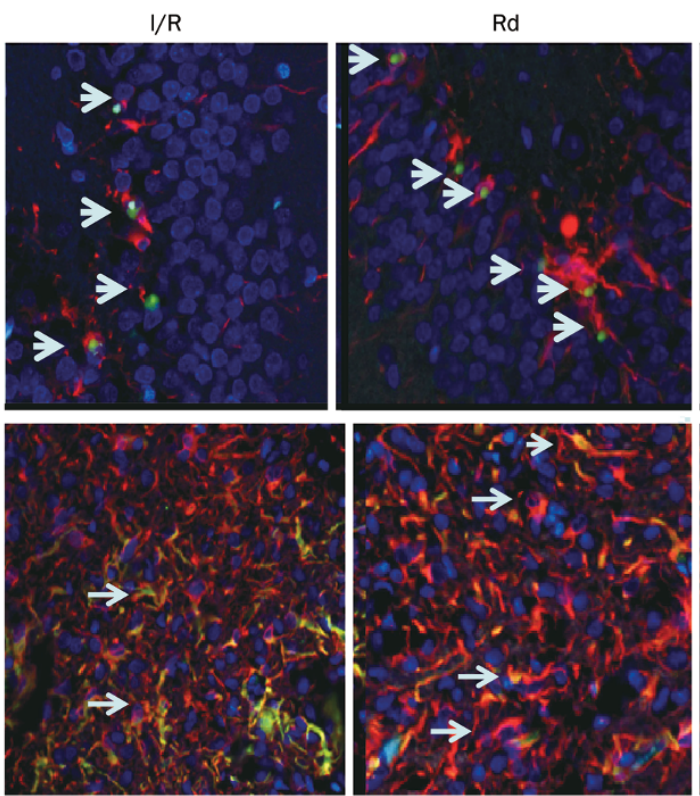

$\mathrm{DCX} / \mathrm{BrdU}(\mathrm{DG})$

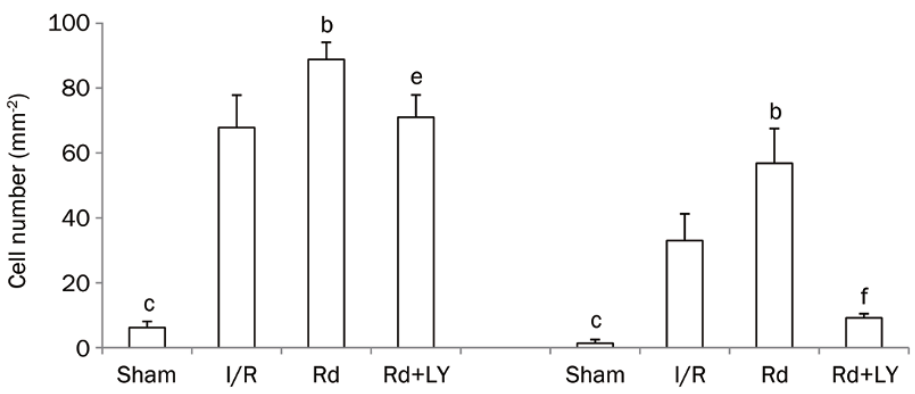

Nestin/GFAP (Str)
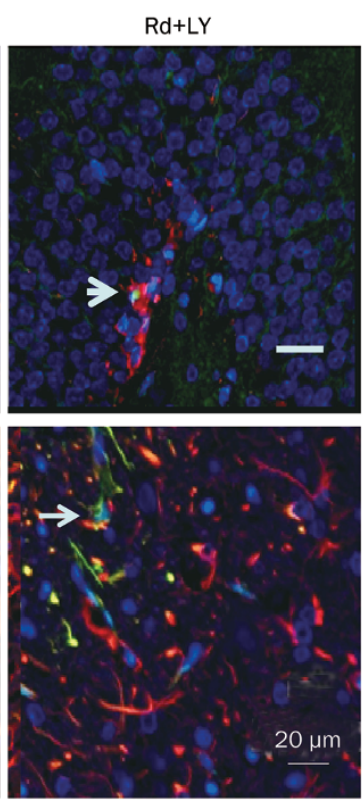
Figure 7. Accumulation of newborn neurons. (A) The merged confocal images confirmed the presence of BrdU (red), GFAP (red), DCX (green), and
Nestin (green) expressing cells in ipsilateral brain sections. Scale bar $=20 \mu \mathrm{mol} / \mathrm{L}$. (B) Quantitative data of each cell type. Means $\pm S D$. $n=10$. ${ }^{b} P<0.05$, ${ }^{\mathrm{c}} P<0.01$ vs I/R. ${ }^{\mathrm{e} P}<0.05,{ }^{\mathrm{f}} P<0.01$ vs I/R+Rd. Str, Striatum; DG, Dentate gyrus; LY, LY294002.

Rd could activate the phosphorylation of Akt and ERK to protect neurons, and this idea is supported by our results (Figures 2-4).

Taken together, as a traditional Chinese medicine that can strengthen the body's resistance to pathogenic factors, ginsenosides such as Rd may prevent cerebral ischemic injury by promoting neurogenesis and increasing the expression of VEGF and BDNF, at least in part by activating the PI3K/Akt and ERK1/2 pathways.

\section{Acknowledgements}

This work was supported by the National Natural Science Foundation of China (№ 81073087 and 81374036), and the International Project of the Ministry of Science and Technology of China (№ 2011ZX09201-201-09).

\section{Author contribution}

Yong-qiu ZHENG designed the study and drafted the manuscript; Xin-Yu LIU and Xin-yu ZHOU coordinated the experiments ; Jin-cai HOU carried out the animal study; Hua ZHU participated in the experimental design and data analyses; and Wang ZHONG was involved in discussion of the experiments and drafts of the manuscript. All authors read and approved the final manuscript.

\section{References}

1 Gaulden J, Reiter JF. Neur-ons and neur-offs: regulators of neural induction in vertebrate embryos and embryonic stem cells. Hum Mol Genet 2008; 17: 60-6.

2 Zhao J, Zhao Y, Zheng W, Lu Y, Feng G, Yu S. Neuroprotective effect of curcumin on transient focal cerebral ischemia in rats. Brain Res 2008; 1229: 224-32.

3 Sugiura S, Kitagawa K, Tanaka S, Todo K, Matsuoka EO, Sasaki T, et al. Adenovirus-mediated gene transfer of heparin-binding Epidermal growth factor-like growth factor enhances neurogenesis and angiogenesis after focal cerebral ischemia in rats. Stroke 2005; 36: 85964.

4 Chen Y, Sun FY. Age-related decrease of striatal neurogenesis is associated with apoptosis of neural precursors and newborn neurons in rat brain after ischemia. Brain Res 2007; 1166: 9-19.

5 Radad K, Gille G, Liu L, Rausch WD. Use of ginseng in medicine with 
emphasis on neurodegenerative disorders. J Pharmacol Sci 2006; 100: 175-86.

6 Shang YH, Tian JF, Hou M, Xu XY. Progress on the protective effect of compounds from natural medicines on cerebral ischemia. Chin J Nat Med 2013; 11: 588-95.

7 Ye RD, Kong XW, Yang QZ, Zhang YX, Han JL, Li P, et al. Ginsenoside $\mathrm{Rd}$ in experimental stroke: superior neuroprotective efficacy with a wide therapeutic window. Neurotherapeutics 2011; 8: 515-25.

8 Wang YM, Jin KL, Mao XO, Xie L, Banwait S, Marti HH, et al. VEGFOverexpressing transgenic mice show enhanced post-ischemic neurogenesis and neuromigration. J Neurosci Res 2007; 85: 740-7.

9 Rosell A, Morancho A, Navarro-Sobrino M, Martinez-Saez E, Hernández-Guillamon M, Lope-Piedrafita S, et al. Factors secreted by endothelial progenitor cells enhance neurorepair responses after cerebral ischemia in mice. PLoS One 2013; 8: e73244.

10 Schäbitz WR, Schwab S, Spranger M, Hacke W. Intraventricular brainderived neurotrophic factor reduces infarct size after focal cerebral ischemia in rats. Cereb Blood Flow Metab 1997; 17: 500-6.

11 Kim YR, Kim HN, Ahn SM, Choi YH, Shin HK, Choi BT. Electroacupuncture promotes post-stroke functional recovery via enhancing endogenous neurogenesis in mouse focal cerebral ischemia. PLoS One 2014; 9: e90000.

12 Sun FY, Guo X. Molecular and cellular mechanism of neuroprotection by vascular endothelial growth factor. J Neurosci Res 2005; 79: 1804.

13 Yan XB, Hou HL, Wu LM, Liu J, Zhou JN. Lithium regulates hippocampal neurogenesis by ERK pathway and facilitates recovery of spatial learning and memory in rats after transient global cerebral ischemia. Neuropharmacology 2007; 53: 487-95.
14 Zhang X, Shi M, Bjørås M, Wang W, Zhang GY, Han JL, et al. Ginsenoside Rd promotes glutamate clearance by up-regulating glial glutamate transporter GLT-1 via PI3K/AKT and ERK1/2 pathways. Pharmacology 2013; 4: 1-8.

15 Hoehn BD, Palmer TD, Steinberg GK. Neurogenesis in rats after focal cerebral ischemia is enhanced by indomethacin. Stroke 2005; 36 : 2718-24.

16 Chen XH, Chen SQ, Jiang Y, Zhu CS, Wu AM, Ma XM, et al. Minocycline reduces oxygen-glucose deprivation-induced $\mathrm{PC} 12$ cell cytotoxicity via matrix metalloproteinase-9, integrin b1 and phosphorylated Akt modulation. Neurol Sci 2013; 34: 1391-6.

17 Tabakman R, Jiang H, Shahar I, Arien-zakay H, Levine RA, Lazarovici P. Neuroprotection by NGF in the PC12 in vitro OGD model involvement of mitogen-activated protein kinases and gene expression. N Y Acad Sci 2005; 1053: 84-96.

18 Saraswati S, Kumar S, Alhaider AA. $\alpha$-Santalol inhibits the angiogenesis and growth of human prostate tumor growth by targeting vascular endothelial growth factor receptor 2-mediated AKT/mTOR/ P70S6K signaling pathway. Mol Cancer 2013; 147: 1-18.

19 Shin HY, Kim JH, Phi JH, Park CK, Kim JE, Kim JH, et al. Endogenous Neurogenesis and neovascularization in the neocortex of the rat after focal cerebral ischemia. J Neurosci Res 2008; 86: 356-7.

20 Wang Y, Galvan V, Gorostiza O, Ataie M, Jin K, Greenberg DA. Vascular endothelial growth factor improves recovery of sensorimotor and cognitive deficits after focal cerebral ischemia in the rat. Brain Res 2006; 1115: 186-93.

21 Bramham CR, Messaoudi E. BDNF function in adult synaptic plasticity: the synaptic consolidation hypothesis. Prog Neurobiol 2005; 76: 99-125. 